Relations industrielles

Industrial Relations

\title{
Executive Compensation in Large Industrial Corporation, by Wilbur G. Lewellen, National Bureau of Economic Research, Columbia University Press, New York, 1968, 371 pages.
}

\section{René Parenteau}

\section{Volume 23, numéro 4, 1968}

URI : https://id.erudit.org/iderudit/027971ar

DOI : https://doi.org/10.7202/027971ar

Aller au sommaire du numéro

Éditeur(s)

Département des relations industrielles de l'Université Laval

ISSN

0034-379X (imprimé)

1703-8138 (numérique)

Découvrir la revue

Citer ce compte rendu

Parenteau, R. (1968). Compte rendu de [Executive Compensation in Large Industrial Corporation, by Wilbur G. Lewellen, National Bureau of Economic Research, Columbia University Press, New York, 1968, 371 pages.] Relations industrielles / Industrial Relations, 23(4), 704-705.

https://doi.org/10.7202/027971ar

Tous droits réservés (C) Département des relations industrielles de l'Université Laval, 1968
Ce document est protégé par la loi sur le droit d'auteur. L'utilisation des services d’Érudit (y compris la reproduction) est assujettie à sa politique d'utilisation que vous pouvez consulter en ligne.

https://apropos.erudit.org/fr/usagers/politique-dutilisation/ 
Papers on Absenteism, by K.N. Vaid, Shri Rom Centre for Industrial Relations, India, 1967, 109 pp.

Au moment où les conditions physiques de travail, les soins médicaux et les revenus s'améliorent, on devrait s'attendre à une diminution des absences dans les entreprises industrielles et les organismes publics. De fait, il appert que le taux d'absentéisme est moins élevé que dans le passé. Cependant, cette baisse ne semble pas se manifester dans les entreprises de l'Inde, en particulier, dans l'industrie du textile. C'est là la raison qui a incité K.N. Vaid et le Centre Shri Ram à entreprendre des études sur l'absentéisme industriel et les facteurs psycho-sociologiques qui peuvent l'expliquer.

Le volume est une collection de quatre articles déjà publiés dans une revue spécialisée en relations industrielles. Les formules utilisées pour calculer le taux d'absentéisme diffèrent radicalement de celles présentées dans les meilleurs volumes en administration du personnel. En fait, l'auteur utilise quatre formules différentes pour les fins de son analyse.

Un dépouillement préliminaire des données permet de conclure qu'un petit groupe d'individus s'absentant de façon chronique fait gonfler le taux d'absence. Les procédures et les règlements concernant l'obtention des permissions de s'absenter constituent un autre déterminant de l'absentéisme.

Pour connaitre les facteurs psycho-sociologiques qui peuvent influencer la décision de s'absenter de son travail, on a construit et administré un questionnaire d'attitudes ò deux groupes différents de travailleurs: ceux qui s'absentent régulièrement et ceux qui sont assidus au travail. L'étude comparative des données montre que les travailleurs qui s'absentent régulièrement partagent moins les objectifs de l'entreprise, sont moins intégrés à leur groupe de travail, sont moins satisfaits de la supervision que ceux qui sont assidus ou travail.

Les causes plus profondes de l'absentéisme sont ajourées par une série d'entrevues auprès de ceux qui s'absentent régulièrement. Le contenu des entrevues a permis au chercheur d'établir cina profils de travailleurs qui s'absentent de façon chronique.

1.-L'entrepreneur: ce type très énergique et original dans ses idées est prêt à assumer des tôches directoriales que l'entreprise ne peut lui offrir.
2.-Le «status seeker »: ce type est un mécontent qui constate le décalage existant entre le statut dont il jouit dans l'entreprise et le standing qu'il croit que la société lu: attribue.

3-- "L'épicurien»: c'est le type qui re cherche un statut social et un revenu élevé mais n'est pas prêt à fournir l'effort néces. saire pour combler ses aspirations.

4.-Le « family man »: c'est le type qui est préoccupé par les problèmies de sa fo. mille au point où il se sent obligé de s'absenter de son travail pour assurer une présence au foyer.

5.-Le type âgé et malade: ce dernier s'absente pour des raisons de maladie chrnnique ou encore à cause d'une constitution psysique débile.

On peut reprocher ò l'auteur le choix des termes pour qualifier le genre de travoilleurs qui s'absentent régulièrement. On peut trouver fastidieux la lecture de nomb.ix tableaux qui apparaissent dans son dernier article. Cependant, c'est un travail de recherche que doivent lire les préposés au personnel dans les entreprises; les étudionts et professeurs en relations industrielles pour les raisons suivantes: a) la démarche utilisée est rigoureuse et elle s'accompagne d'un appareil statistique très simple, facile is saisir; b) les résultats de la recherche peuvent servir de fondements solides à l'élebnration d'une politique d'embauche et de formation du personnel; c) la bibliogrophie imposante qui opparaît à la fin du volume contient les meilleurs ouvrages publiés $5 \mathrm{Jr}$ l'absentéisme depuis plusieurs années

\section{Lourent BELANGER}

\section{Executive Compensation in Large Industrial} Corporation, by Wilbur $\mathrm{G}$. Lewellen, $\mathrm{Na}$ tional Bureau of Economic Resecrch, Columbia University Press, New-York, 1968, 371 pages.

Ce n'est pas tous les jours qu'une itide scientifique nous permet d'entrer dans les niveaux supérieurs de l'administration de: grandes corporations. Celle-ci le fait pour étudier d'une part la rémunération, d'cutre part les différentes formes de compensation pour les directeurs de compagnies. En effet, l'étude de Lewellen a porté sur 250 directeurs de compagnies, soit $5 \mathrm{mem}$ bres de l'exécutif de 50 grandes firmes 
La raison du choix des directeurs de compagnies pour fonder une telle étude est simple; un tel choix permet d'abord d'éclaircir une situation devenue de plus en plus embrouillée, de comprendre pourquoi et dans quelle mesure la rémunération des directeurs a fait place à de multiples formes de compensation. Ce choix permet ensuite de soisir l'impact des taxes et de l'impôt sur l'augmentation du revenu réel des salariés les plus fortement rémunérés dans l'économie. Enfin, une telle étude limitée à des directeurs de compagnies fait intervenir un outre problème cuisant, celui de la motivation même des directeurs, ce groupe dont l'intéressement a une portée si grande sur le développement des économies nationales.

Les conclusions et résultats de recherche de Lewellen étonnent. Pour éviter les charges écrasantes de l'impôt progressif, les directeurs ont opté pour d'autres formes de compensation que les salaires et bonus; en 1963, par exemple, moins de la moitié de la compensation après la taxe des directteurs était composée des salaires et bonus. Ces outres formes de compensation (actions préférentielles, paiements différés, bénéfices de pension, plans de partage des profits) non seulement permettent aux directeurs de jouir d'exemption de taxes, mais aussi leur accordent une nouvelle position stratégique dans l'entreprise égale à celle des entrepreneurs et des actionnaires privilégiés.

En outre, les résultats statistiques sont ainsi regroupés qu'ils permettent d'établir une évolution historique de la compensation des directeurs depuis la guerre, une comparaison entre les compagnies, une comparaison entre la compensation des cadres inférieurs et celle des cadres supérieurs de I'industrie.

La partie méthodologique de la recherche nous résume toutes les formes et tous les plans d'exemption de taxes et d'avantages supplémentaires des directeurs de compagnies; la partie empirique de l'analyse nous donne la mesure et l'extension qu'ont pris ces plans. Enfin, six appendices ajoutent à cette information six analyses détaillées concernant l'utilisation de plans particuliers et les techniques utilisées pour calculer le bénéfice total.

\section{René PARENTEAU}

The Industrial Composition of Income and

Product, by John W. Kendrick, editor, National Bureau of Economic Research,

Columbia University Press, New-York, 1968, 494 pages.
Cet ouvrage contient les textes exposés à la « Conference on the Industrial Composition of Income and Product tenue en décembre 1966. Ces textes représentent des études basées sur de nouveaux estimés rapportés dans les comptes nationaux. Ces nouveaux estimés rendaient possible l'analyse des changements survenus dans la structure économique car ils présentaient en dollars courants et constants le produit national par industrie et des tables comparatives entre l'investissement et la production, entre le revenu et la dépense, car ils présentaient en somme la valeur ajoutée produite par chaque industrie.

Toute étude qui part de ces nouveaux estimés peut donc s'avérer d'un grand intérêt non seulement pour analyser les changements passés dans les structures économiques, mais aussi pour prévoir des mouvements futurs... n'est-ce pas là une autre démarche importante vers la planification du développement économique. Outre une étude nouvelle de l'économie nationale, toute recherche en ce sens peut maintenant analyser quantitativement des phénomènes non purement économiques du développement économique, tels les changements technologiques, I'innovation, la requalification et l'utilisation nouvelle de la main-d'oeuvre. Voilà les principales qualités des trois études présentées dans ce recueil.

Première partie: "Changes in Final Demand, Industry Product, and Prices $\gg$. Cette première étude cherche ò expliquer les changements des prix survenus entre les années 1958-1964, à analyser les causes des changements de la composition du produit réel au cours des années 1947-1958. La première onalyse, celle d'Alterman, étudie les changements des prix surtout en fonction des variations de la valeur ajoutée; elle développe également l'utilisation des tables * revenu-dépense » pour expliquer les changements des prix. La seconde analyse explique les changements dans la production industrielle; les auteurs, Vaccara et Simon, concluent que le principal facteur de ces phénomènes est la demande plus que les changements des coefficients techniques.

Deuxième partie: "Changing Factor Costs and Shares of Cross Income by Industry $\mathbf{x}$. Greenberg and Mark étudient d'abord les différences des coûts des facteurs par industrie selon deux critères, les gains moyens par heure de travail et la production par homme-heure. Ils comparent ensuite les fructuations entre les années 1947-1960 et les expliquent par le niveau moyen comparé de l'emploi, par lo technicité particulière à 